\title{
PECULIAR CASE
}

or

\section{GELATINIFORM CANCER,}

IN WHICH NEARLY ALL THE ORGANS OF THE BODY CONTAINED COLLOID TUMOURS ;

WITH THE APPEARANCES ON DISSECTION.

By JOHN C. WARREN, M.D., PROPESSOR OF ANATOMY AND BURGERY IN BATARD UNIVERSITY.

Communicated by MARShaLL HALL, M.D., F.R.S. S. L. and Ed.

READ JUNE $25 \mathrm{TH}, 1844$.

Collord, gelatinoma, or gelatiniform cancer, as it has been called, is stated by the distinguished authors who have described this disease, as occurring in one single mass, usually of considerable size. Dr. Walshe, in his work on cancer, says, "In the description of scirrhus, the comparative rarity of the co-existence of several tumours in the same subject has been noted; this is still more marked in the case of colloid. M. Cruveilhier states, that it is rare to observe its successive or simultaneous development in a number of organs or parts. Dr. Hodgkin expresses himself nearly in the same tone, but adds, that it does at times invade different localities in the same subject. It may co-exist with the two other species of carcinoma, in distinct organs." vOL. XXVII. $2 \mathrm{C}$ 
In the case here described the disease was diffused through nearly all the textures of the body without presenting any one considerable mass.

This case is also remarkable in exhibiting a union of the three admitted forms of malignant disease, scirrhoma, cephaloma, and gelatinoma. I must observe, however, that my opportunities of examining the colloid form of malignant affection have been few, compared with the other two forms. Perhaps those who are more versed in its pathology may even doubt the propriety of enrolling this under the head of gelatinoma. I have stated the facts as accurately as I could, and leave the character of the disease to be decided by those who are better able to do so.

There seems to me to be an obscurity in the discrimination of the forms of malignant disease, which requires for its dissipation a great number of new observations, made by accurate and experienced pathologists.

J. B., a painter by trade, residing in Boston, not married, twenty-five years of age, applied to me in May 1840, on account of a tumour upon the right side of the neck.

In his family history there did not appear any thing peculiar : his father, being forty-eight years of age, died of inflammation of the lungs; his mother was still living, in good health; two brothers and two sisters were also living, in health; he had lost one brother by measles in early life. He himself had measles, and afterwards jaundice, when very young; his health from the time of recovery to the 
present has been good, with the exception of an intermittent fever at New Orleans about three years since, from which he recovered on leaving the city ; he has been able to work, until within a month.

At the time of the application his personal appearance was emaciated and pallid; he complained of loss of appetite. The tumour, for which he applied, had existed for about a year and a half, and was situated on the right side of the neck, immediately above the clavicle. It was rather hard, slightly moveable, and presented, on the whole, the appearance of a scrofulous enlargement of the lymphatic glands, except that it was harder than those glands usually are when thus affected. There were also one or two other tumours upon the trunk, which had appeared within a month, and one upon the left arm, which may be described as a type of the others, situated on the anterior and middle part of the upper arm. It had a bluish white colour. The skin which covered it was perfectly natural as to colour, and was moveable on the surface of the tumour. It was somewhat elastic, about the size of a nutmeg, not painful, nor sensitive to the touch, and had, on the whole, a slight resemblance to a limited varicose tumour.

Presuming his disease to be of a scrofulous character, I recommended the internal use of the tincture of iodine, and an ointment to be applied externally containing the hydriodate of soda, four leeches to the tumour of the neck, to leave his business, and go into the country.

2 c 2 
After the lapse of ten days he came to me again, calling himself better : the tumour in his neck was a little diminished : the continuance of the same remedies was therefore advised to him. Ten days more having expired, he came again to me, when I found that the tumour in the neck had continued to diminish, but his general health was not so good. I therefore advised him to omit the external use of the hydriodate of soda, but to continue the internal use of the tincture of iodine, and to make a fresh application of leeches to the tumour.

On the 20th of June he entered the Massachusetts General Hospital, under the care of Dr. Townsend ; and, as his case was interesting, I continued to observe him carefully through the different phases of the disease which presented themselves. At this time his general health did not appear very much affected; his pulse and tongue indicated no great disturbance ; the appetite was tolerable; but there was some oppression after taking food. The tumour of the neck was about the size of a hen-egg; there were also a number of tumours scattered over the surface, especially of the abdomen, generally about the size of a hazel nut, many of them, however, not being larger than small shot, irregularly rounded in form, elastic to the touch. The skin was perfectly white over them, the bluish tint appearing to emanate from the internal tumour through the transparent skin. At my request $\mathrm{Dr}$. Townsend opened one of these tumours, on doing which, after the skin was cut, a delicate membrane was observed, 
covering a little cluster of pearl-like granulations, containing a gelatinous substance and some watery fluid. A small vein ran into the tumour, ramifying freely over the surface of the cells, and from this issued venous blood, which continued to flow for a few minutes.

The day subsequent to his admission to the hospital he began to complain of severe pain in the abdomen and back, which finally limited itself to the left iliac region and left hypochondrium, and after several days was much relieved by the application of leeches. Pain in the head also commenced, while he yet laboured under the pain of the abdomen, say ten days subsequent to the origin of the latter. It continued to advance in severity for some days, until the left eye was affected, the conjunctiva being considerably injected: by suitable applications this was relieved, and for a day or two he seemed comparatively comfortable, the pain in the head being less. The respite, however, was only for a couple of days, when the pain in the head again returned, and continued to increase in severity till it became very violent, all treatment affording only a temporary relief.

Upon the eighteenth day of this second accession, and the fifty-third of his admission to the hospital, the pulse was very variable, from 68 to 100 ; his appearance resembling that of a man under the influence of opium, being stupid, and roused with difficulty. These appearances were ascribed to the opiate he had taken, but without good reason, as it appeared 
subsequently; for, after the lapse of a fortnight, during most of the time being more or less stupid, he said that he had double vision with the right eye, there being some dilatation of the pupils, with strabismus. In a degree this was relieved previous to his discharge, which took place, at the request of his friends, on the 2nd of September, his condition not being improved. The tumours, with no variation, except a slight increase in size and modification of colour, being darker, had now increased to a number between thirty and forty, covering the abdomen and limbs.

After his return home he lingered till the 21 st of September, gradually sinking, without any material change in his condition, excepting that he was delirious for two days before death.

Autopsy. - In the examination of the body, which took place eight hours after death, I was aided by the excellent pathologist Dr. J. B. S. Jackson, a number of other gentlemen being present, among whom were Drs. Townsend, Adams, of Waltham, and J. M. Warren.

External appearance.-The body was emaciated; its surface studded with subcutaneous tumours, generally about the size of a hazel nut, of a bluish colour, and of slight elasticity to the touch.

Tumours.-Several were removed and opened; they were composed of small granulations, constituted by sacs containing a substance which appeared at first view to be wholly gelatinous, but which, on being divided, discharged a small quantity of viscid 
fluid. The colour was a mixed grey and red ; they were slightly transparent ; in consistence they were friable : most of them were connected with small veins, and had a vascular tissue upon their outside. Many of the lymphatic glands were indurated, especially the inguinal, which exhibited very large absorbent vessels running in and out of them. The tumour of the neck had not varied in size, and consisted of a yellowish white, moderately firm, elastic substance; in colour, resembling the deposit sometimes seen in scrofulous glands, but in consistence, different from that of these glands, in having a greater degree of firmness.

The thyroid gland was enlarged, hardened, and exhibited small gelatiniform bodies.

Head.-The cranium being cut into, displayed a number of the minute, semi-transparent, gelatinous tumours in the diploë of the frontal and occipital regions. Near the superior angle of the occipital bone was a spot, about half an inch in extent, which reached quite through, so as to adhere to the dura mater; also another, which did not extend through, existed upon the temporal bone. The excavation in the bones was filled by small pearl-like granulations, of a gelatinous consistence.

The membranes investing the brain were dry and opaque: the brain itself appeared dry; its vessels, particularly of the cineritious part, were dark-coloured. In the ventricles were from 4 to 5 ounces of serum; the plexus choroïdes was pale, and without tumours. Thorax.-The tumours were found in the muscles, 
both internal and external to them; they also occupied the ribs, to such an extent as to render some of them quite soft. A considerable number existed in the mediastinum, the pleura itself being quite healthy.

The heart, externally, was covered with gelatiniform bodies, generally about half the size of a pea; some were larger. These tubercles were most abundant in the course of the principal arteries and veins. The muscular substance of the auricles and ventricles presented a considerable number of the gelatinous formations. In the cavity of the left auricle were found two gelatiniform bodies, of a globular form, adhering to the internal parietes of the auricle. These, examined by the microscope, were seen to be composed of minute granules, barely visible by a glass magnifying thirty times, but by the aid of the most powerful simple lens, these granules appeared to contain others. The coagulum found entangled in the mitral valves had somewhat the same appearances under the same magnifying powers.

The lungs appeared, at the time of the autopsy, to be healthy, but could not then be minutely examined. On a subsequent examination, a small gelatiniform tumour was noticed on the surface of the left lung, and on cutting into these organs, a number of similar bodies were found sparingly scattered throughout their texture; in other respects, these organs were somewhat hyperæmied, but for the rest in a perfectly healthy state. The bronchial 
glands were hardened, and exhibited the same appearance as the tumour of the neck.

Abdomen.-The absorbent vessels upon the surface of the abdominal muscles contained an opaque substance, which struck us at the time to be of a cerebriform character; but whether it was really encephaloid matter, or the opaque form of the gelatinous substance, which existed in other parts of the body, could not be certainly ascertained. The omentum was free of fat, and of tumours. The mesentery was studded with the tumours, and the mesenteric glands were enlarged: one just above the right inguinal canal was as large as an English walnut: the whole of them containing the same whitish opaque substance as the tumour of the neck. There were a number of these tumours about the pyloric orifice of the stomach; but the texture of the stomach itself was healthy, and had no gelatiniform appearances.

The liver was greatly diseased, being filled with the tubercles, which projected above the surface. These tubercles, when cut into, appeared of a yellowish white colour, and granulated texture; they were destitute of the transparency which characterized the tumours throughout the body, and closely resembled the appearances of scirrhoma of the liver depicted by Carswell. The substance of the liver itself was dark.

The pancreas was much enlarged and hardened, of a rounded oblong form : there were some of the tumours on its surface. The spleen was not altered. 
Kidneys.-These organs had in their interior a number of the gelatiniform tumours, particularly the right kidney. There were many of them in regular masses, contained in the cellular substance surrounding the kidneys. The supra-renal capsules were enlarged and hardened.

Pelvic organs. - The bladder was free from disease.

The testes were enlarged, and in each of them two or three indurated, opaque, whitish tumours might be found.

The thoracic duct was larger than natural at the lower part, and was compressed above by a number of diseased glands.

The arteries and veins were thin and pale, and exhibited no appearance of redness in their internal coat.

The blood was small in quantity; thin, watery, and of a dark colour: no gelatiniform bodies were detected in it.

The appearance of the muscles covering the thoracic cavity has been already described. Many of the muscles, in other situations, presented on their surface and in their substance, a great number of gelatiniform bodies, varying in size from that of a pea to that of granules not distinctly visible without the aid of a microscope. Some of the muscles seemed to be almost composed of these bodies, particularly the gracilis muscle of the thigh and the rectus muscle of the abdomen, which were carefully dissected, and a portion of them preserved. 
Remarks.-The appearances in this case, as already stated, were peculiar, and not exactly accordant with those which $I$ have seen described in any case that I am acquainted with. The texture of the large tumour of the neck, and that of various lymphatic and mesenteric glands, considerably resembled the common scrofulous tubercles of these glands.

The lungs, which, according to M. Louis, are always found to contain tubercles of a scrofulous character, when these exist in any part of the body, formed a remarkable exception in this peculiar disease, to the condition of nearly all the other organs.

The appearances in the liver were such as are seen connected with scirrhoma; but the vast number of tumours found in most of the organs of the body were of quite a different description. They bore a decided resemblance, in texture, to what has been described by Cruveilhier, Velpeau, and other distinguished pathologists, under the name of Gelatiniform Cancer. They differ, so far as I know, from all the descriptions which have been given of this latter affection, in being small and dispersed through all the organs of the body; whereas this disease is described, by the best pathologists, to be, in most cases, concentrated in one continuous mass. Whether these considerations would lead them to rank this disease as a variety of gelatiniform cancer, or as one of a different and anomalous character, I must leave to them to decide. 
It was very curious to observe these tumours in all the various degrees of development, from the point of their deposition and formation to their attainment of a considerable magnitude. Whatever was their size, the same transparent granular appearance was always visible, there being no difference except in the size of the transparent granules. The opaque whitish substance was confined altogether to the system of lymphatic glands.

Among so many varieties in form and stage of this affection, I was not without the hope of discovering some morbid changes, which might have been supposed to have preceded the formation of the gelatiniform substance. But no anterior preliminary change in the state of the cellular, muscular, vascular, or any other texture could be discovered, either by the eye, or the microscope.

Specimens of the disease from various parts of the body were preserved, and subjected to many subsequent examinations. Contrary to the opinion expressed by some authors in regard to gelatiniform tumours, all these tumours soon lost their transparency on immersion in alcohol, and presented the same yellowish white appearance as the diseased lymphatic glands.

This case goes to support the opinion of eminent pathologists, namely, of Carswell, Laennec, Otto, Cruveilhier, Müller, and of Walshe, (whose valuable work on Cancer has been recently republished in this country under the direction of Dr. J. M. Warren,) of the identity of the three forms of malig- 
nant disease, Scirrhoma, Cephaloma, and Gelatinoma. The scirrhomatous appearance was seen in the liver, and in some of the lymphatic glands; the cephalomatous in some of the lymphatic vessels; and the gelatinomatous in almost every part of the body.

In a fatal case of gelatinoma, which I have met with since this case occurred, two of the appearances indicated above were distinctly combined, namely, extensive gelatiniform disease in the large intestine joined to a scirrhomatous state of the neighbouring mesaraic glands. 